\title{
An Image-based Dynamic High-throughput Analysis of Adherent Cell Migration
}

Meng Sun ${ }^{1, *}$, Bence Rethi ${ }^{1}$, Akilan Krishnamurthy ${ }^{1}$, Vijay Joshua ${ }^{1}$, Heidi Wähämaa ${ }^{1}$, Sergiu-Bogdan Catrina $^{2}$ and Anca Catrina ${ }^{3, *}$

${ }^{1}$ Rheumatology Unit, Department of Medicine, Karolinska Insititutet, Stockholm, Sweden; ${ }^{2}$ Department of Molecular Medicine and Surgery, Karolinska Institutet, Stockholm, Sweden; ${ }^{3}$ Rheumatology Unit, Department of Medicine, Karolinska University Hospital Center for Rheumatology, Academic Specialist Centre, Stockholm, Sweden

*For correspondence: anca.catrina@ki.se; meng.sun@ki.se

[Abstract] In this protocol, we describe a method to monitor cell migration by live-cell imaging of adherent cells. Scratching assay is a common method to investigate cell migration or wound healing capacity. However, achieving homogenous scratching, finding the optimal time window for end-point analysis and performing an objective image analysis imply, even for practiced and adept experimenters, a high chance for variability and limited reproducibility. Therefore, our protocol implemented the assessment for cell mobility by using homogenous wound making, sequential imaging and automated image analysis. Cells were cultured in 96-well plates, and after attachment, homogeneous linear scratches were made using the IncuCyte ${ }^{\circledR}$ WoundMaker. The treatments were added directly to wells and images were captured every 2 hours automatically. Thereafter, the images were processed by defining a scratching mask and a cell confluence mask using a software algorithm. Data analysis was performed using the IncuCyte ${ }^{\circledR}$ Cell Migration Analysis Software. Thus, our protocol allows a time-lapse analysis of treatment effects on cell migration in a highly reliable, reproducible and re-analyzable manner. Keywords: Cell migration, Scratching assay, Live cell image, Time-lapse imaging, High-throughput

[Background] Scratching assays are a widely used method for investigating cell migration or wound healing capacity. However, the conventional method (manual scratching) requires skill to perform linear scratches and is an end-point assay (Liang et al., 2007; Krishnamurthy et al., 2016). Data are usually manually analyzed with ImageJ or other software. Recently, we employed a high-throughput automatic imaging system, IncuCyte ZOOM from Essen Bioscience, in a cell migration assay (Sun et al., 2019). By using IncuCyte ${ }^{\circledR}$ WoundMaker, linear scratches can be created homogeneously in up to 96 -wells at the same time. With the appropriately defined algorithm, by analysis of phase-contrast, cell confluence masks and scratching masks, cell migration can be simultaneously evaluated. In brief, the conventional method is more laborious and time-consuming than the method we present here. This protocol provides a method with minimized time and effort for processing high-throughput samples and analyzing data in an unbiased way over time. 


\section{Materials and Reagents}

1. IncuCyte ${ }^{\circledR}$ ImageLock 96-well Plates (Essen Bioscience, catalog number: 4379)

2. Synovial fibroblast (Isolated from RA patients undergoing joint replacement, Sun et al., 2019)

3. Normal human dermal fibroblasts (PromoCell, catalog number: C-12300)

4. Primary Human Osteoarthritis Synovial Fibroblasts (Bioivit, catalog number: HPCSFOA-03)

5. Dulbecco's Modified Eagle Medium (DMEM) (Sigma-Aldrich, catalog number: D5796-500ml)

6. Fetal bovine serum (FBS) (Sigma-Aldrich, catalog number: F7524)

7. Trypsin-EDTA (Sigma-Aldrich, catalog number: T3924-100ml)

8. Phosphate buffered saline (PBS) (Sigma-Aldrich, catalog number: D8537-500ml)

9. Penicillin-streptomycin (PEST) (Sigma-Aldrich, catalog number: P4333-100ml)

10. Anti-citrullinated protein antibody (Purified from peripheral blood of RA patients, Ossipova et al., 2014)

11. Recombinant Human TNF- $\alpha$ (Peprotech, catalog number: $300-01 \mathrm{~A}$ )

12. Recombinant Human IL-8/CXCL8 Protein (R\&D Systems, catalog number: 208-IL-010)

13. Alconox powder (VWR, catalog number: 21835-123)

14. Sachets, Rely+On ${ }^{T M}$ Virkon ${ }^{\circledR}$ powder (VWR, catalog number: 148-0200)

15. Sterile distilled water (produced in house)

16. $70 \%$ ethanol ( Sigma-Aldrich, catalog number: $470198-1 \mathrm{~L}$ )

17. Synovial fibroblasts culture medium (10\% FBS) (see Recipes)

18. Starvation medium (serum free) (see Recipes)

19. Low-serum cell culture medium (2\% FBS) (see Recipes)

\section{Equipment}

1. IncuCyte ${ }^{\circledR}$ WoundMaker with two wash boats (Essen Bioscience, catalog number: 4493)

2. IncuCyte ZOOM live-cell analysis system (Essen Bioscience, model: IncuCyte ${ }^{\circledR}$ ZOOM$)$

3. Multi-Channel pipette, 8-channel, 20-200 $\mu \mathrm{l}$ (VWR, Ergonomic High Performance Multichannel Pipettor, catalog number: 89079-948)

\section{Software}

1. Cell Migration Analysis Software Module (Essen Bioscience, catalog number: 4400)

2. Prism 6 (GraphPad Software)

3. IncuCyte ${ }^{\circledR}$ Zoom software (2018A)

\section{Procedure}

\section{A. Prepare Cells}


1. Seed Cells in $150 \mu \mathrm{l}$ complete growth medium in a 96-well imagelock plate using a multi-channel pipette at a cell density that will reach up to $95 \%$ confluence overnight. In 96 -well plates, it is advisable to exclude the outer wells from the experiment due to evaporation effects.

Note: We seed 20,000 synovial fibroblasts or human dermal fibroblasts per well to reach full confluence within $24 \mathrm{~h}$.

2. Fill the outer wells with $300 \mu \mathrm{l}$ PBS to counteract evaporation-effects in rest of the wells.

3. Grow cells at $37^{\circ} \mathrm{C}$ in a humidified incubator with $5 \% \mathrm{CO}_{2}$ overnight or until cells reach $95 \%$ confluence.

4. Wash cells with $100 \mu \mathrm{l}$ PBS twice using a multi-channel pipette.

5. Starve cells with $100 \mu \mathrm{l} \mathrm{FBS} \mathrm{free} \mathrm{culture} \mathrm{medium} \mathrm{in} \mathrm{a} \mathrm{humidified} \mathrm{incubator} \mathrm{with} 5 \% \mathrm{CO}_{2}$ for $2 \mathrm{~h}$ to deplete growth factors.

Note: In our setting, we starve cells for $2 \mathrm{~h}$. Overnight starvation is commonly used for growth factor depletion. Starvation time may differ, depending on the experimental setting.

B. Make scratch

1. Clean the wound maker in the wash boat for 5 minutes each in a series of four wash solutions (45 ml of each)
a. $0.5 \%$ Alconox
b. $1 \%$ Virkon
c. sterile distilled water
d. $70 \%$ ethanol

2. Use the IncuCyte ${ }^{\circledR}$ Wound Maker to create homogenous scratches (https://www.youtube.com/watch?v=x7pMzJ1VIdA\&feature=youtu.be)
a. Remove top of the wound maker and place it in an empty wash boat.
b. Insert plate into base plate holder and remove plate cover.
c. Replace pin block by guiding the rear dowels of pin block into the rear holes of the base plate.
d. Push and hold the black lever.
e. Lift pin block while continuing to hold the black lever down.

3. Discard the medium using multi-channel pipette without disturbing the scratch.

4. Add $200 \mu \mathrm{l}$ of Low-serum culture medium (2\% FBS) containing the experimental treatments. Note: We treat our synovial fibroblasts or human dermal fibroblasts with anti-citrullinated protein antibodies (ACPA) (Ossipova et al., 2014), control lgG, Tumor necrosis factor (TNF) and medium only.

\section{IncuCyte Zoom Scan setup (Figure 1A and Figure S1)}

To avoid interrupting an ongoing scan, it is very important to check device status. Place a new plate only between scheduled scans. Do not eject door during scanning (Figure S2).

1. Place 96-well Imagelock plate and click 'Schedule Scans'. 
2. Select the tray position of interest.

3. Click 'add vessel' and select '96-well Essen Imagelock' in Zoom software.

4. Set scan type to 'Scratch Wound' with 'Wide Mode' and 'Scan pattern'.

5. Select 'Phase contrast' channels in Zoom software and Set plate layout as desired.

6. Select the desired scan frequency and timing by, right clicking on the time base and selecting 'Set Interval'. Scan interval depends on how many wells need to be scanned. We recommend a 1-2 $\mathrm{h}$ interval for a migration assay.

7. Click 'Apply' button to finish setting. Any unapplied changes will not be performed.

D. Plate Setup (optional) (Figure 1B)

1. Under 'properties' panel, enter 'label', 'cell type', 'passage' and 'notes'. In a high throughput assay, it is advisable to keep detailed information about experiments for further reference.

2. Click 'plate map' and select 'add' in dropdown list to create assay layout. It is highly recommended to use plate map for further analysis.

3. In plate map editor, click 'NEW' to create treatment.

4. Select wells in the plate layout, enter concentration or dilution of treatment and add to selected wells.

5. Apply step 4 to all treatments and complete plate map.

6. Click 'OK' button to save plate map.

\section{A. Incucyte Zoom Scan setup}

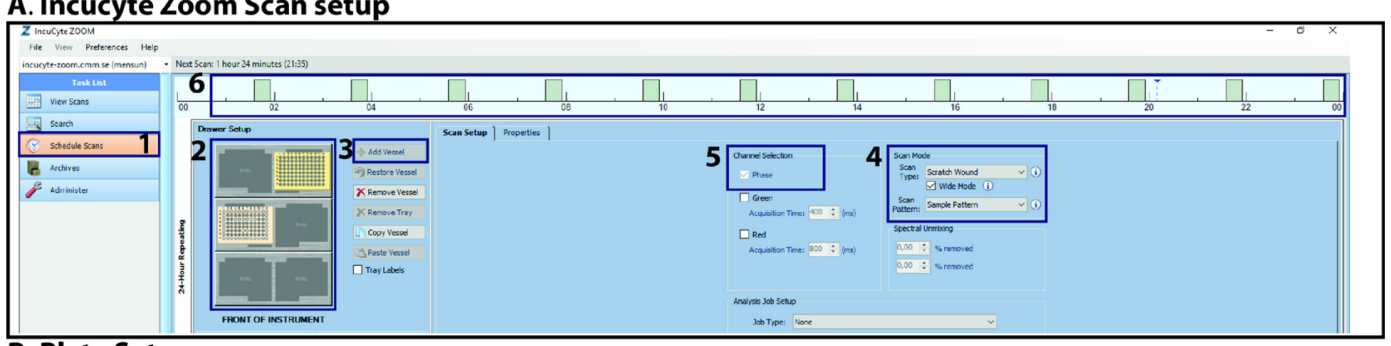

B. Plate Setup

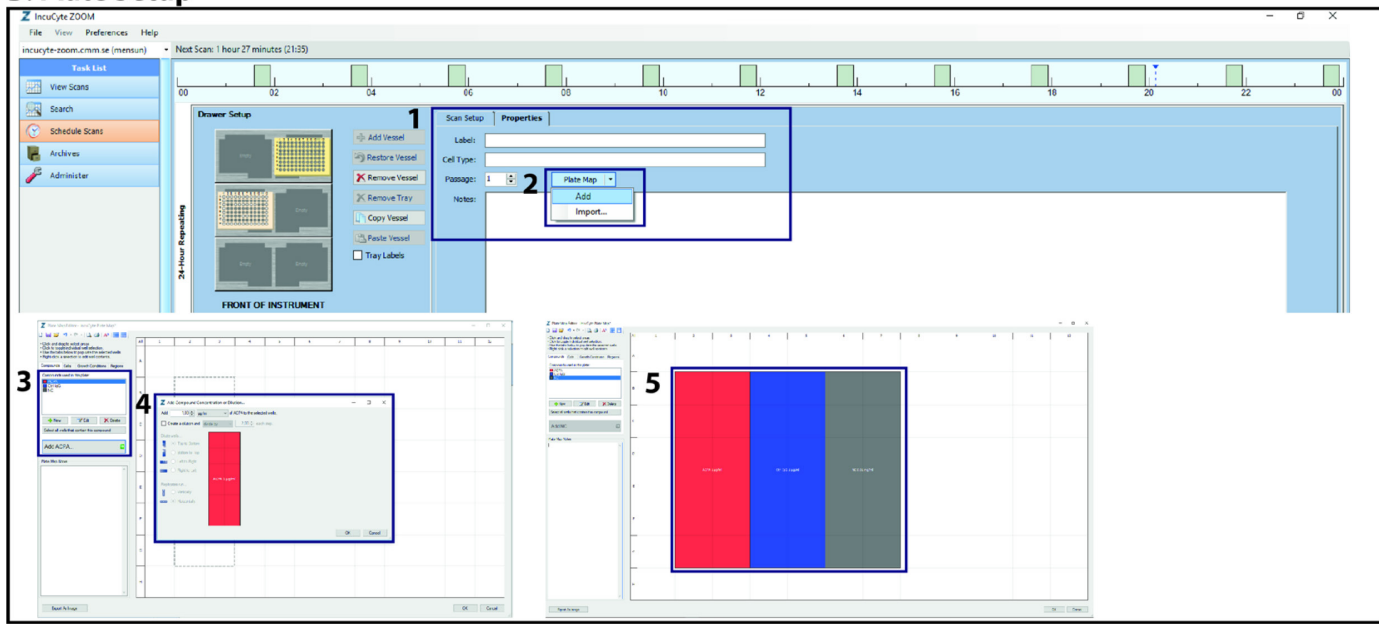

Figure 1. An illustration of scan and plate setup for using IncuCyte Zoom system. Screenshots of the IncuCyte ZOOM software for Scan setup and Plate setup. A. Steps 1 to 6 
show the procedure to setup plate position, scan mode, channel and intervals for scan setup.

B. Steps 1 to 5 show the procedure to setup experiment properties, plate layout and treatment information.

\section{E. Data Collection and Processing}

1. In side menu, select the experiment that needs to be analyzed.

2. Create or Add image collection (Figure 2A).

a. In 'analysis job utilities', click 'Create or Add image collection'.

b. Select several images to create image collection for algorithm definition. It is recommended to select from different locations from plate layout and different time points from scan time to represent whole experiment.

c. Save the image collection with appropriate title.

3. New processing definition (Figure $2 \mathrm{~B}$ ).

a. In 'analysis job utilities', click 'New processing definition'.

b. Select the newly created image collection and continue to define the scratching mask.

c. In left side menu, drag slide bar towards either 'background' or 'cell' to adjust segmentation.

d. In clean up panel section, enter a certain number to fill up the holes between cells (optional).

e. In filter section, select 'min' in 'Area' and enter a number to exclude cell debris in the image.

f. Select 'phase contrast' in Image Channel, 'scratching wound mask' and 'confluence mask' in Analysis Mask.

g. Click 'preview' to check the current processing definition and click 'preview all' to apply current definition to all images in collection.

h. Adjust the definition until it fits most of images in collection and save it with a proper name.

i. The processing definition can be applied for batch experiments.

4. Launch Analysis Job (Figure 2C).
a. In 'analysis job utilities', click 'Launch Analysis Job'.
b. In pop-up window, enter the name of the analysis.
c. Select start and finish time point to define time range.
d. Select wells in plate layout and click 'Launch' to start analysis job.
e. Check analysis result in main menu in 'search' and under 'analysis job' tab.

F. Data Export (Figure 2D)

1. In side menu, select the experiment that needs to be exported.

2. Select 'Metrics' panel, choose appropriate 'phase metrics' and click 'Graph/Export' button.

3. In pop-up window, select 'wells' and 'time points/time range' and click 'Data Export' button.

4. In 'Export Metrics' window, select 'Layout', 'Destination', 'Other option' and Click 'Export' button to export data for further analysis. 

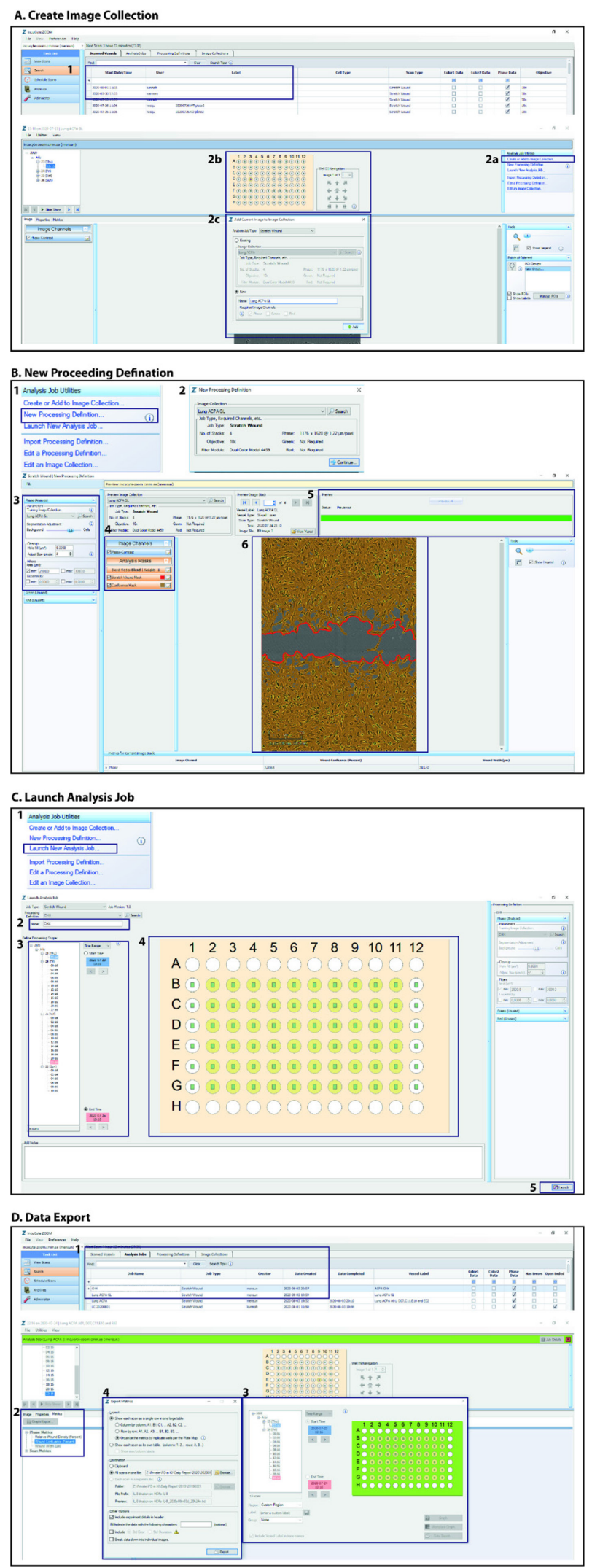

Figure 2. An illustration of data processing for using Cell Migration Analysis Software Module. Screenshots of the cell migration analysis software for data processing. A. Steps to select re-presentive images from all timepoints to create image collection. B. Steps to define 
confluence mask and wound mask for images from collection. C. Steps to launch analysis for selected plate and desired time range. D. Steps to export raw data.

\section{G. Lens Changing (Figure S3)}

IncuCyte Zoom offers three different optical (4×, 10x, and 20x lens). To perform migration assay, it is essential to have $10 \times$ lens. To minimize the risk of conflict, changing lens is only available via administer account.

1. In main menu, go to 'Task list', click 'administer' and select tab 'optical configuration'.

2. Follow the steps of 'optics configuration' to install new lens.

3. Accept the change and create new schedule.

\section{Data analysis}

1. Representive example of data and confluence mask segmentation.

When the analysis job is done, scratch wound mask, confluence mask and initial scratch wound mask are obtained at all timepoints. The wound confluence is simultaneously calculated by IncuCyte ${ }^{\circledR}$ Cell Migration Analysis Software. An example is shown in Figure 3: the confluence mask segmentation at the initial time point, end time point (Figure $3 \mathrm{~A}$ ) and wound confluence curve in time lapse, where cells were treated with antibodies: A, B and control antibody (Figure 3B).
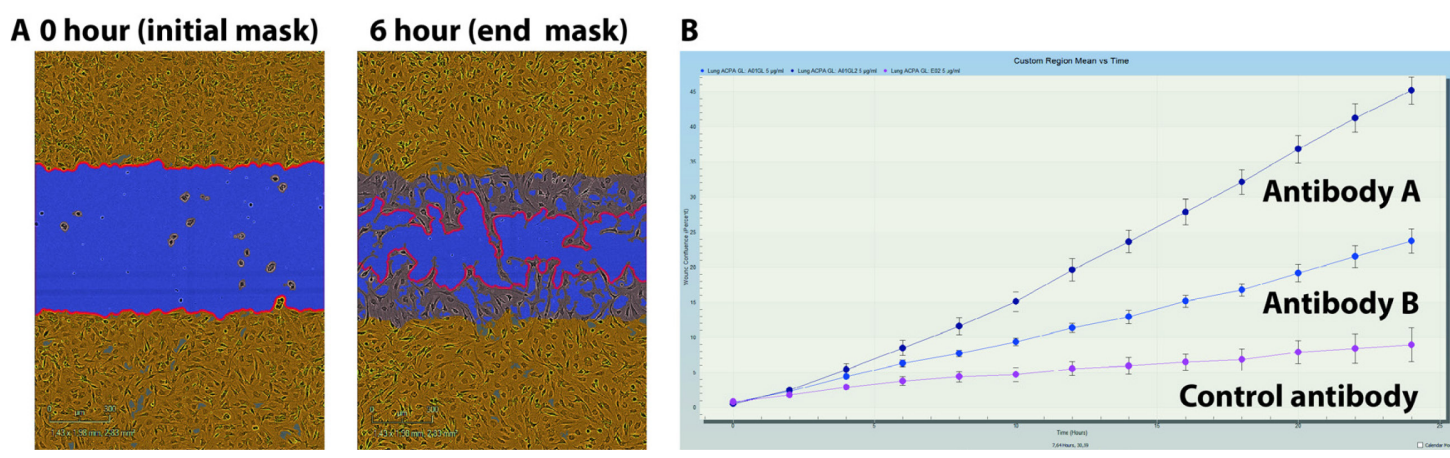

Figure 3. Example of confluence mask, scratch mask and analysis of wound confluence using IncuCyte Cell migration analysis software. Exported images from IncuCyte analysis software. A. A segmentation example of cell confluence mask (yellow) and scratch mask (red outline) at $0 \mathrm{~h}$ (initial time point) and $6 \mathrm{~h}$ (end point). B. An example of migration rate curve (wound confluence) between 0 to $24 \mathrm{~h}$, where antibody $\mathrm{A}$ and $\mathrm{B}$ but not control antibody have effect on cell migration.

2. Data analysis

Incucyte Zoom software allows data export. The default figure of migration rate (percentage of wound confluence) is good enough for overview results. However, for statistical analysis, it is recommended to export raw data to Prism. Moreover, it is very useful to extract data and 
compare migration rates at specific time points. In Figure 4, we show migration rate, where fibroblasts were treated with ACPA, Ctrl IgG or medium only [The figure was originally published in Sun et al. (2019), Figure 1, as an open access article distributed in accordance with the Creative Commons Attribution 4.0 Unported (CC BY 4.0) license (https://creativecommons.org/licenses/by/4.0/)]. Data from same wells, with or without serum starvation, were analyzed for migration up to $20 \mathrm{~h}$ (Figures 4A and 4B) and images of the wound mask at time point $6 \mathrm{~h}$ is shown in Figure $4 \mathrm{C}$. Data were normalized to the medium-treatment group at time point $6 \mathrm{~h}$ and presented as migration fold change (Figures $4 \mathrm{D}$ and $4 \mathrm{E}$ ). We also performed cell migration assays on both human dermal fibroblasts (HDFs) and synovial fibroblasts of osteoarthritis patients (OASFs) with or without stimulation of IL-8 or TNF-alpha (Sun et al., 2019, Supplementary Figure 2).
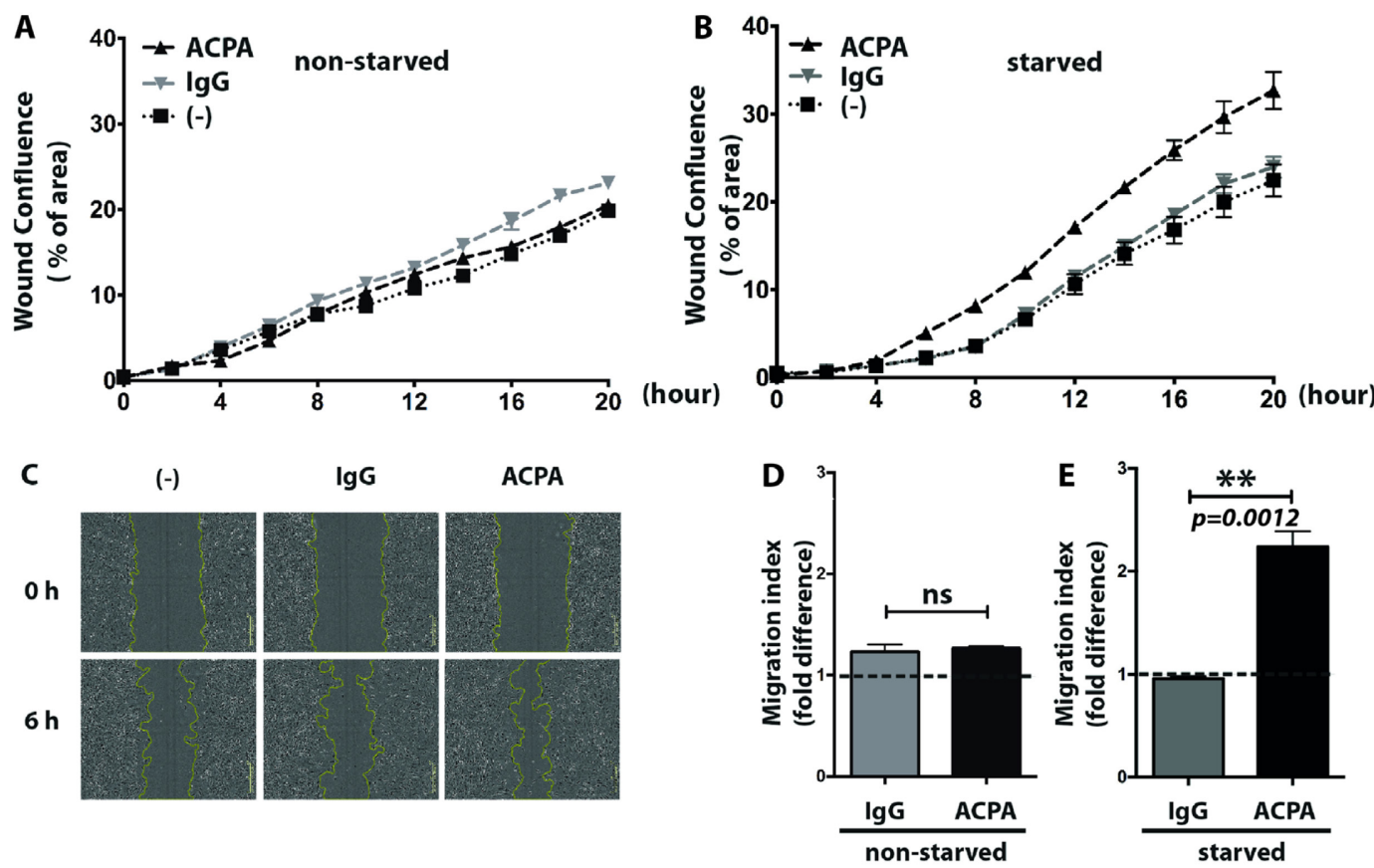

Figure 4. Increased mobility of synovial fibroblasts in the presence of polyclonal ACPAs.

Real-time cell migration was measured in the presence of $1 \mu \mathrm{g} / \mathrm{ml}$ ACPA, control $\mathrm{lgG}$ or without any treatment in non-starved (A) and starved (B) fibroblast cultures using IncuCyte. Imagebased evaluation of cell migration in starved fibroblast cultures were performed using Cell Migration Analysis software module with 10x magnification (C). Cell mobility was analyzed during a period of $6 \mathrm{~h}$ in the presence of $1 \mu \mathrm{g} / \mathrm{ml}$ polyclonal ACPA IgGs (ACPA) or non-ACPA control IgGs (IgG) or without antibody treatment (-) in both non-starved (D) and starved (E) fibroblast cultures. Dot line indicate migration index of non-treated fibroblasts. The graphs represent mean \pm SD values of 6 replicates for each treatment. ${ }^{*} P<0.05$. 


\section{$\underline{\text { Recipes }}$}

1. Synovial fibroblasts culture medium (10\% FBS)
a. Dulbecco's Modified Eagle Medium (DMEM $500 \mathrm{ml}$ )
b. Add $50 \mathrm{ml}$ heat-inactivated fetal bovine serum (FBS) to reach $10 \%$
c. Add $100 \mathrm{U} / \mathrm{ml}$ penicillin
d. Add $100 \mu \mathrm{g} / \mathrm{ml}$ streptomycin

2. Starvation medium (serum free)
a. Dulbecco's Modified Eagle Medium (DMEM 500ml)
b. Add $100 \mathrm{U} / \mathrm{ml}$ penicillin
c. Add $100 \mu \mathrm{g} / \mathrm{ml}$ streptomycin

3. Low-serum cell culture medium ( $2 \%$ FBS)
a. Dulbecco's Modified Eagle Medium (DMEM 500ml)
b. Add $10 \mathrm{ml}$ heat-inactivated fetal bovine serum (FBS) to reach $2 \%$
c. Add $100 \mathrm{U} / \mathrm{ml}$ penicillin
d. Add $100 \mu \mathrm{g} / \mathrm{ml}$ streptomycin

\section{Acknowledgments}

This project has received funding from FOREUM, Foundation for Research in Rheumatology, from the European Research Council (ERC) under the European Union's Horizon 2020 research and innovation program (grant agreement CoG 2017 - 7722209_PREVENT RA and grant agreement 777357_RTCure), from the Swedish Research Council and Konung Gustaf V:s och Drottning Victorias Frimurarestiftelse.

Last, we would like to pay our gratitude and our respects to our group leader and colleague, Prof. Anca Catrina who passed away recently. She was a dedicated professor and excellent researcher in the Department of Medicine, Rheumatology Unit, at Karolinska University Hospital. We will continue her work and her style as much as we can

The protocol was first described in the methods section of Sun et al. (2019).

\section{Competing interests}

The authors declare that they have no conflicts of interest.

\section{Ethics}

This study involves human participants with ethical permit listed below:

1. Kartläggning av prediktiva biomarkörer vid kronisk artrit ID: 2009-358-31-3. (Mapping of predictive biomarkers in chronic arthritis). 
2. Kartläggning av inflammatoriska mediatorers betydelse för sjukdomsförlopp vid kroniskaledsjukdomar ID:2009-1262-31-3. (Mapping of inflammatory mediators significance for disease course in chronic joint diseases).

\section{References}

1. Krishnamurthy, A., Joshua, V., Haj Hensvold, A., Jin, T., Sun, M., Vivar, N., Ytterberg, A. J., Engstrom, M., Fernandes-Cerqueira, C., Amara, K., Magnusson, M., Wigerblad, G., Kato, J., Jimenez-Andrade, J. M., Tyson, K., Rapecki, S., Lundberg, K., Catrina, S. B., Jakobsson, P. J., Svensson, C., Malmstrom, V., Klareskog, L., Wahamaa, H. and Catrina, A. I. (2016). Identification of a novel chemokine-dependent molecular mechanism underlying rheumatoid arthritis-associated autoantibody-mediated bone loss. Ann Rheum Dis 75(4): 721-729.

2. Liang, C. C., Park, A. Y. and Guan, J. L. (2007). In vitro scratch assay: a convenient and inexpensive method for analysis of cell migration in vitro. Nat Protoc 2(2): 329-333.

3. Ossipova, E., Cerqueira, C. F., Reed, E., Kharlamova, N., Israelsson, L., Holmdahl, R., Nandakumar, K. S., Engstrom, M., Harre, U., Schett, G., Catrina, A. I., Malmstrom, V., Sommarin, Y., Klareskog, L., Jakobsson, P. J. and Lundberg, K. (2014). Affinity purified anticitrullinated protein/peptide antibodies target antigens expressed in the rheumatoid joint. Arthritis Res Ther 16(4): R167.

4. Sun, M., Rethi, B., Krishnamurthy, A., Joshua, V., Circiumaru, A., Hensvold, A. H., Ossipova, E., Gronwall, C., Liu, Y., Engstrom, M., Catrina, S. B., Steen, J., Malmstrom, V., Klareskog, L., Svensson, C., Ospelt, C., Wahamaa, H. and Catrina, A. I. (2019). Anticitrullinated protein antibodies facilitate migration of synovial tissue-derived fibroblasts. Ann Rheum Dis 78(12): 1621-1631. 